\title{
ARE MENTAL BLOCKS FORGOTTEN \\ DURING CREATIVE PROBLEM SOLVING \\ DUE TO INHIBITORY CONTROL?
}

\author{
A Thesis \\ by \\ GENNA MARIE ANGELLO
}

\begin{abstract}
Submitted to the Office of Graduate Studies of Texas A\&M University

in partial fulfillment of the requirements for the degree of

MASTER OF SCIENCE
\end{abstract}

August 2011

Major Subject: Psychology 
Are Mental Blocks Forgotten

During Creative Problem Solving

Due to Inhibitory Control?

Copyright 2011 Genna Marie Angello 


\title{
ARE MENTAL BLOCKS FORGOTTEN \\ DURING CREATIVE PROBLEM SOLVING \\ DUE TO INHIBITORY CONTROL?
}

\begin{abstract}
A Thesis
by

GENNA MARIE ANGELLO

Submitted to the Office of Graduate Studies of Texas A\&M University

in partial fulfillment of the requirements for the degree of

MASTER OF SCIENCE
\end{abstract}

Approved by:

Chair of Committee, Steven M. Smith

Committee Members, Darrell Worthy

Julie Linsey

Head of Department, Ludy T. Benjamin

August 2011

Major Subject: Psychology 


\author{
ABSTRACT \\ Are Mental Blocks Forgotten \\ During Creative Problem Solving \\ Due to Inhibitory Control?
}

(August 2011)
Genna Marie Angello, B.A., University of California, Los Angeles Chair of Advisory Committee: Dr. Steven M. Smith

Attempting to retrieve a target from memory via a retrieval cue can cause competition from the cue's associates, which might block the target. A 1994 study by Anderson, Bjork, and Bjork demonstrated retrieval-induced forgetting for competing associates and suggested that inhibitory control resolving competition causes the forgetting. A 2011 study by Storm, Angello, and Bjork found forgetting for incorrect associates following creative problem solving. This thesis investigated whether such forgetting is also the result of inhibitory control. Competition was manipulated by instructing participants to remember or forget incorrect associates before working on a Remote Associates Test problem. If problem-solving-induced forgetting is caused by inhibition, then to-be-remembered associates should suffer more forgetting than to-beforgotten associates.

Overall, forgetting occurred for incorrect associates participants were instructed to remember and forget. However, the first quartile of trials showed forgetting only for 
to-be-remembered associates following longer problem solving durations, suggesting a possible role of inhibitory control as an active means to overcome fixation in creative problem solving. 


\section{TABLE OF CONTENTS}

Page

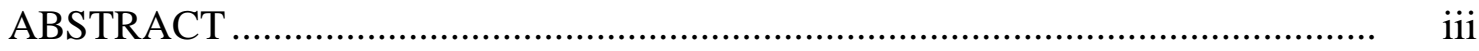

TABLE OF CONTENTS …................................................................ v

LIST OF FIGURES ......................................................................... vi

1. INTRODUCTION

1.1 Blocks in Creative Problem Solving ............................................ 1

1.2 Forgetting Fixation ................................................................. 3

1.3 Retrieval Inhibition ............................................................. 6

2. PROBLEM

2.1 Testing for Inhibitory Control in Creative Problem Solving ............. 16

2.2 Method ................................................................................... 19

\section{RESULTS AND DISCUSSION}

3.1 Overall Results .................................................................... 26

3.2 Exploratory Analysis ........................................................... 28

4. GENERAL DISCUSSION ..................................................................... 31

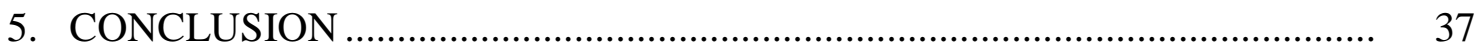

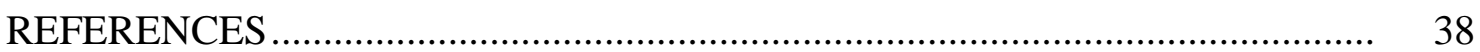

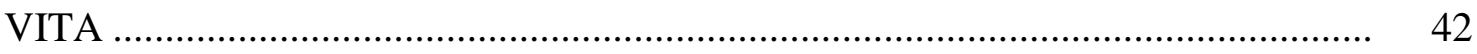




\section{LIST OF FIGURES}

FIGURE $\quad$ Page

$1 \quad$ Diagram of Trial Sequence .......................................................... 15

2 Predicted Results Using Inhibitory Control Explanation ....................... 18

3 Predicted Results Using Non-Inhibitory Explanation ........................... 19

$4 \quad$ Sequence of Current Study's Trials ..................................................... 21

$5 \quad$ Overall Results Separated by Within-Subjects Condition ...................... 27

6 First Quartile Results Separated by Within-Subjects Condition .................. 30 


\section{INTRODUCTION}

\subsection{Blocks in Creative Problem Solving}

Productive thinking plays an important role in creativity and allows people to move beyond current knowledge and skills in order to create innovative ideas or products. In contrast, reproductive thinking involves knowing how to apply knowledge toward a problem to generate the solution. Although productive thinking involves looking past a current framework or mental set, oftentimes it is adaptive to retain certain features of the current framework rather than reject the entire thing (Weisberg, 1995).

One type of creative problem that involves mentally searching through a current framework to make a novel combination was created by Mednick (1962). Mednick's Remote Associates Test (RAT) problems each present three seemingly unrelated words (e.g., bass, complex, and sleep). To solve the problem, the participant must respond with a solution word (e.g., deep) that is associated independently with each of the three problem words. The solution word can be a synonym (e.g., deep, complex) or part of a commonly spoken phrase with one of the presented words (e.g., "deep sleep"), or it can share a more general semantic relation with the problem word (e.g., deep, bass). While searching for these "remote associates," however, other associates (and possibly, the correct remote associate) come to mind. For example, one might associate guitar with the word bass, hard with complex, and rest with sleep. Focusing on these incorrect associates would prevent the answer, deep, from coming to mind, creating a mental

This thesis follows the style of The Quarterly Journal of Experimental Psychology. 
block. Such mental blocks in RAT problem solving have been induced experimentally and have resulted in impaired performance (Smith \& Blankenship, 1991).

Smith and Blankenship (1991) hypothesized that if participants focus on incorrect associates during RAT problem solving, they will be led down the wrong solution path, away from the correct answer. Additionally, the participants will fixate on the incorrect associates, thus keeping themselves on the wrong solution path. Participants in the fixation group were presented with misleading associates, such as leg, furnace, and pear, for each of three RAT problem words, such as arm, coal, and peach, whereas participants in the non-fixation group were shown only the RAT problem words. Those in the fixation group were told that the presented associates were examples of solution words, but were not actual solutions. Participants in the fixation group performed significantly worse than those in the non-fixated group, yielding a fixation effect. Even when fixation was induced prior to RAT problem presentation via a paired associates learning task to prime memory for the misleading associates, Smith and Blankenship found a similar fixation effect. Each learned pair, such as arm leg, contained a RAT problem word and the misleading associate. Sixty such pairs, corresponding to every problem word presented in the 20 RAT problems, were learned and immediately tested. Subsequent RAT performance was significantly worse for the fixation condition compared to the non-fixation condition, which received a paired associates learning task with pairs such as arm election, comprised of RAT problem words and unrelated misleading associates, prior to problem solving. 


\subsection{Forgetting Fixation}

Various forms of fixation can block solutions to problems. Smith (2003) lists forms such as "typical thinking, implicit assumptions, and recent experience." Smith explains that recent experience can create a biased retrieval set when the first-retrieved items take away from the shared retrieval strength of related items and reduce the likelihood of retrieval for any additional items (e.g., Smith \& Vela, 1991). Smith and Vela predicted that discontinuity between two different memory searches is adaptive for forgetting fixation and accessing once-blocked items. They manipulated this discontinuity using varying intertest intervals, hypothesizing that larger intertest intervals following the first failed retrieval attempt should result in successful retrieval, or reminiscence, for items that were previously blocked and inaccessible. They reasoned that successful reminiscence results when new initial search probes are used on the second test, and that the intertest interval allows for the forgetting of the old, unsuccessful search probes used during the first test. Supporting their prediction, the greatest reminiscence was found during the first minute of recall following longer intertest intervals, suggesting that participants were returning to the retrieval task with better search probes for accessing blocked items.

If fixation is induced during creative problem solving, it may be possible for participants to forget interfering information, or at least put that interfering information temporarily out of mind, which might release them from fixation. According to the 
forgetting-fixation hypothesis, correct solutions that were previously blocked by interfering items will become accessible following the forgetting of those interfering items (Smith \& Blankenship, 1989). To test their hypothesis, Smith and Blankenship presented subjects with rebus problems, which displayed words in certain formations (e.g., fly night), and the task was to produce the common English phrase that described the words (e.g., "fly by night"). However, during the first presentation of the problem, an incorrect solution (e.g., over) was displayed alongside the problem to cause fixation and block the production of the correct solution. Then, following 0,5 or 15 minutes, participants were re-presented with the block of problems without the incorrect solution. In support of forgetting-fixation, the group of subjects re-presented with the problems after 5 or 15 minutes were more likely to correctly solve the once-blocked problem and were less likely to recall the incorrect solution following problem solving when compared with the control group that received an immediate re-presentation of the block of problems.

Support for forgetting fixation, although not directly measured in recall of misleading associates following delayed problem solving, was found in improved RAT problem solving following a delay for fixated participants (Smith \& Blankenship, 1991). Smith and Blankenship predicted that forgetting interfering items and unblocking correct solutions should only result in improved performance when there are interfering items to be forgotten. Initially presented RAT problems, whose words participants had learned related misleading associates (fixation group) or unrelated misleading associates (nonfixation group), were presented a second time, either immediately or following a 5 
minute delay. Smith and Blankenship measured improvement as increased problem solving success during the second RAT presentation when compared with the problem solving success during the first RAT presentation. Supporting their prediction, they found greater improvement in RAT performance following the delay for the fixation group, when compared with the improvement observed for the non-fixation group.

RAT improvement only for participants that are presented with misleading associates has since been replicated (Vul \& Pashler, 2007; Wiley, 1998). In both Vul and Pashler and Wiley's studies, however, when participants led themselves down the wrong path by self-inducing mental blocks through retrieving incorrect associates to RAT problem words, no improvement occurred following a delay. Interestingly, Vul and Pashler did demonstrate improvement following a delay when participants had been presented with misleading associates that they knew were, in fact, incorrect associates prior to problem solving.

Wiley's (1998) study failed to demonstrate improvement following a delay for participants high in knowledge for the misleading domain even when those participants were presented with the misleading associates in a memory task prior to RAT problem solving. However, Wiley's participants were never informed that the misleading associates were actually incorrect solutions to the RAT problems. Although participants low in the misleading domain knowledge were able to demonstrate improvement following a delay, participants high in the misleading domain knowledge incorrectly applied that knowledge toward solving the RAT problems. However, because no feedback was given regarding the inappropriateness of applying that knowledge, these 
participants most likely saw no reason to forget the misleading associates during the delay. In addition to seeing improved RAT performance following the opportunity to forget fixation when participants have something to forget, it also seems necessary for participants to realize that they are fixated before forgetting fixation can lead to improved RAT performance. This would suggest that forgetting fixation is an active process that allows for the inhibition caused by suppression of interfering information (Wiley, 1998), rather than an indirect side effect of returning to the problem following a delay.

\subsection{Retrieval Inhibition}

Retrieval inhibition is the process of suppressing interfering information, making it less accessible, while encoding or retrieving target information (Anderson \& Bjork, 1994). One observable result of retrieval inhibition is retrieval-induced forgetting (Anderson, 2003; Anderson, Bjork, \& Bjork, 1994; Anderson \& Spellman, 1995). Retrieval-induced forgetting occurs for items related to a retrieval cue that compete for access when the cue is used to guide the retrieval of a target item. Anderson et al. (1994) presented participants with category-exemplar word pairs, such as fruit banana; fruit orange; fruit guava; fruit mango; tree birch; tree elm; tree juniper; tree ash. Participants were asked to study each pair, presented individually, by relating the exemplar to its category. Participants were then asked to practice retrieving half of the exemplars, such as orange and mango, from half of the categories three times each, one- 
at-a-time in a random order, such that neither a given exemplar nor a given category was practiced in succession. During retrieval practice, participants saw the category as a retrieval cue, followed by the first two letters of the target exemplar (e.g., fruit or fruit ma ). Following a 20-minute distractor task, participants were given a surprise cued-recall test for all of the studied exemplars, each presented individually for 10 seconds. During this final recall test, participants were presented with the category as a retrieval cue, followed by the first letter of the target exemplar (e.g., fruit b ). In order to control for output interference, the practiced exemplars were always tested last because it was expected that the retrieval practice would strengthen their accessibility and this strengthened accessibility would be further strengthened during the final test and lead to reduced accessibility for exemplars from the same category (McGeoch, 1942).

The strengthening produced during retrieval practice was, indeed, shown in the final recall performance of Anderson et al.'s (1994) study. The exemplars that had received retrieval practice, although tested last, were recalled more often than nonpracticed exemplars. Retrieval-induced forgetting also occurred on the final test for those non-practiced exemplars, such as banana, that belonged to the practiced categories, when compared with the recall of non-practiced exemplars, such as birch, from non-practiced categories. Additionally, evidence for the forgetting of nonpracticed exemplars from practiced categories on the final test as a result of competition resolution during retrieval practice came from asymmetric retrieval-induced forgetting. Retrieval-induced forgetting occurred only for those non-practiced exemplars strongly 
associated, according to frequency norms, with the practiced category, such as banana, but not for weakly associated non-practiced exemplars, such as guava. This asymmetrical pattern of forgetting was found regardless of the associative strength of the practiced exemplars. Anderson et al. argued that non-practiced exemplars weakly associated with the category do not compete for access during retrieval practice of the target item, and are therefore not subject to the targeted inhibition that results in later forgetting for the strong associates that are more likely to compete for access during retrieval practice. Since retrieval inhibition appears to target those items that interfere and compete for retrieval access, it is possible, then, that a selective retrieval inhibition process can also act to push away irrelevant information that is strongly related to information in a problem, such as the common or stereotypical incorrect associates that are retrieved during RAT problem solving.

Motivated by such a possibility, Storm, Angello, and Bjork (2011) tested for problem-solving-induced forgetting following RAT problem solving. The experimental procedure was similar to that of Anderson et al. (1994); with the substitution of RAT problem solving for category-cued retrieval practice, as well as learning and later taking a memory test for RAT problem words paired with incorrect associates rather than category-exemplar pairs. Participants first studied associated cue-response pairs of moderate forward associative strength, such as board wood; magic trick; death die; shopping buy; picture painting; washer clothes, by relating the response, second, word to the cue, first, word. Following study, participants were shown each cue word and asked to provide the associated response word. This cued-recall test continued until the 
participant had successfully recalled each response word. Then, participants solved 10 RAT problems, such that each problem was presented for a total of 40 seconds while the participant attempted to verbally generate the correct solution. If the participant was successful before the 40 seconds had elapsed, however, the participant was presented with the next problem. Additionally, participants were given feedback so that every time they responded with an incorrect associate, they were informed that this was not the correct answer and they should continue to solve the problem while it remained on the screen.

Each presented RAT problem from Storm et al.'s (in press) study was comprised of three previously studied cue words (e.g., board, magic, death). Participants were informed prior to problem solving that the previously tested response words would never be correct RAT problem solutions (e.g., black). Care was taken to ensure that the associated response words were unrelated to both the problem solutions and to one another. Following a 5-minute distractor task, participants were given a surprise cuedrecall test for all of the originally studied cue-response word pairs. Participants were presented with each cue word for 5 seconds and asked to provide the associated studied response word. Half of the tested pairs served as baseline pairs because they each consisted of cue words that were never presented during problem solving, while the remaining half consisted of cue words that also served as RAT problem words. Final recall performance for response words associated with RAT problem words, critical response words, was compared with performance of baseline response words. Problemsolving-induced forgetting was predicted for critical response words, when compared 
with baseline response words, due to these words competing for retrieval access during RAT problem solving, potentially causing fixation, and becoming suppressed by retrieval inhibition. Supporting this prediction, participants showed forgetting for associates paired with RAT problem words following problem solving compared to recall for associates that were paired with RAT problem words that were never presented during problem solving.

Evidence to support a connection between overcoming fixation during RAT problem solving and retrieval-induced forgetting was reported by Storm and Angello (2010) with a positive correlation between the amount of retrieval-induced forgetting participants exhibited on a cued-recall test following retrieval practice and subsequent RAT problem solving performance following the study of misleading associates, unrelated to the words used to test for retrieval-induced forgetting, paired with the RAT problem words. These results suggest that both problem-solving-induced forgetting and retrieval-induced forgetting occur due to a retrieval inhibitory mechanism that acts to push away the non-target, unwanted items that compete for retrieval access because they share an association with the cue that is present during problem solving or retrieval practice, in the form of the RAT problem word or the category cue.

A second observable result of retrieval inhibition is found in studies of directed forgetting (e.g., Bjork, 1970). According to Bjork, Bjork, and Anderson (1998), directed forgetting, or the instruction to forget previously presented items, induces a motivation to forget unwanted items and focus on wanted and relevant items. Directed forgetting instructions result in retrieval inhibition that is a temporary suppression of items that 
interfere during learning or retrieval of related items. Bjork (1989) reasoned that the forget instructions must be working to reduce recall accessibility of to-be-forgotten items, consequently making a second set of items more accessible. Importantly, the first set of items are only forgotten when they are given forget instructions following presentation and when a second set of related items are subsequently studied (Gelfand \& Bjork, 1985). This supports the conception of retrieval inhibition as a goal-directed process that facilitates retrieval by reducing competition from interfering items (Postman, Stark, \& Fraser, 1968) and also requires an active process such as learning or retrieving related items (Anderson \& Bjork, 1994). Anderson (2005) notes that a possible difference between inhibitory mechanisms of directed forgetting and retrievalinduced forgetting may lie in the actual target of the temporary suppression. For instance, directed forgetting paradigms such as that of Bjork (1970), in which the forget instructions follow the presentation of a first set of items prior to the presentation of a second set of related items, induces forgetting that targets the first set context, rather than specific presented items of the first set. In contrast, retrieval practice within a retrieval-induced forgetting paradigm (Anderson et al., 1994) acts to induce forgetting that targets the specific item that competes for retrieval access via the retrieval practice cue.

Alternative explanations of retrieval-induced forgetting that do not require an inhibitory mechanism include: associative bias, cue bias, and executive control bias models (Anderson \& Bjork, 1994). The associative bias model attributes forgetting of the competing items to increased associative strength between the cue and the retrieved 
target item, which prevents the cue from later accessing the competing item (McGeoch, 1942). According to the cue bias model, the competing item is forgotten because its retrieval cue is changed to a new cue that no longer acts to access the item. For example, if orange and lemon are retrieved using the cue Fruit, this cue may change in representation to Citrus Fruit and when Fruit is later presented as a cue during the final test, the participant may be unable to think of tropical fruits, such as banana and pineapple, because the Fruit cue has been redefined. The executive control bias model states that the competing item is forgotten at the final test that follows retrieval of the target item because of the particular retrieval strategy used by the subject during the final test. According to this explanation, the retrieval cue may be redefined prior to the final test, but the forgetting is the direct result of the participant choosing to limit his or her search during the final test based on the redefined retrieval cue. For example, suppose the Fruit cue is redefined to be mentally represented as Citrus Fruit, during the final test the participant will stop the search much sooner compared to a participant who still includes tropical fruits in the Fruit cue's definition. In this case, the forgetting is not due to inaccessibility but due to the time at which retrieval search is terminated.

The problem with these three non-inhibitory explanations of retrieval-induced forgetting is that they can not account for the forgetting that has been shown to be competition-dependent (for a review see Storm, 2011). Storm, Bjork, and Bjork (2007) manipulated competition during retrieval practice using directed forgetting. They hypothesized that if studied non-practiced exemplars from practiced categories are given forget instructions prior to retrieval practice they will not compete for retrieval access 
because they will already be suppressed due to directed forgetting. Accordingly, if retrieval-induced forgetting is, in fact, the result of inhibitory control that acts to resolve competition during retrieval practice by suppressing interfering competitors, retrievalinduced forgetting should not occur for exemplars given directed forgetting instructions. Whereas, non-practiced exemplars from practiced categories that participants are instructed to remember prior to retrieval practice should compete during retrieval, and thus, should be subject to inhibitory control and exhibit retrieval-induced forgetting following retrieval practice. An alternative prediction, based on the non-inhibitory explanations of retrieval-induced forgetting attributes the forgetting of non-practiced exemplars from practiced categories to interference from strengthened practiced exemplars, re-defined category cues, or biases in retrieval strategies on the final test (Anderson \& Bjork, 1994). According to these non-inhibitory explanations, retrievalinduced forgetting should still occur for non-practiced exemplars from practiced categories, regardless of whether these items are given forget or remember instructions.

During each trial (Figure 1), Storm et al. (2007) presented participants with a list of four category-exemplar pairs, such as fruit apple; flower tulip; fruit kiwi; flower lily. Each list presented two exemplars for two categories. Participants were instructed to study each list, relating each exemplar to its category, because they might be given a memory test for the four pairs. After 8 seconds, the list left the screen and participants were given remember or forget instructions. If they were instructed to remember the previously presented list, they were told that there was a 60 percent chance that they would be tested on the pairs they just saw so that they would be shown the categories 
(e.g., fruit , flower fruit , flower ) and they would be asked to respond with the studied exemplars. If they were instructed to forget the previously presented list, they were told that there was a 0 percent chance that they would be tested on the pairs they just saw. Following the instructions, participants completed retrieval practice for exemplars that were never presented but belonged to one of the presented categories using category cues followed by the first two letters of the target exemplar (e.g., fruit ch fruit ma fruit le , fruit gu

). After retrieval practice on 60 percent of the trials including remember instructions, participants were tested on the studied exemplars. During the remaining trials, half including remember instructions and half including forget instructions, participants were not tested on the studied exemplars. The order of trials was randomized so that participants could not predict the type of instructions they would be given. After all of the trials were presented, participants completed a 5-minute distractor task. Then, participants were given a surprise category-plus-one-letter-stem-cued recall test for all of the studied exemplars, including those they were earlier instructed to forget. The exemplars that had been tested during the remember trials were tested last during the final test and were not included in the critical analysis of retrieval-induced forgetting.

Supporting the role of inhibitory control in resolving competition during retrieval practice, Storm et al. (2007) found retrieval-induced forgetting for non-practiced, never tested exemplars (e.g., apple) from practiced categories when they were given remember instructions but not when they were given forget instructions. Although an associative, cue, and executive bias could have caused retrieval-induced forgetting for exemplars 
given forget instructions, forgetting was only found for exemplars that competed during retrieval of the target exemplar because they belonged to the same category and had been instructed to be remembered.

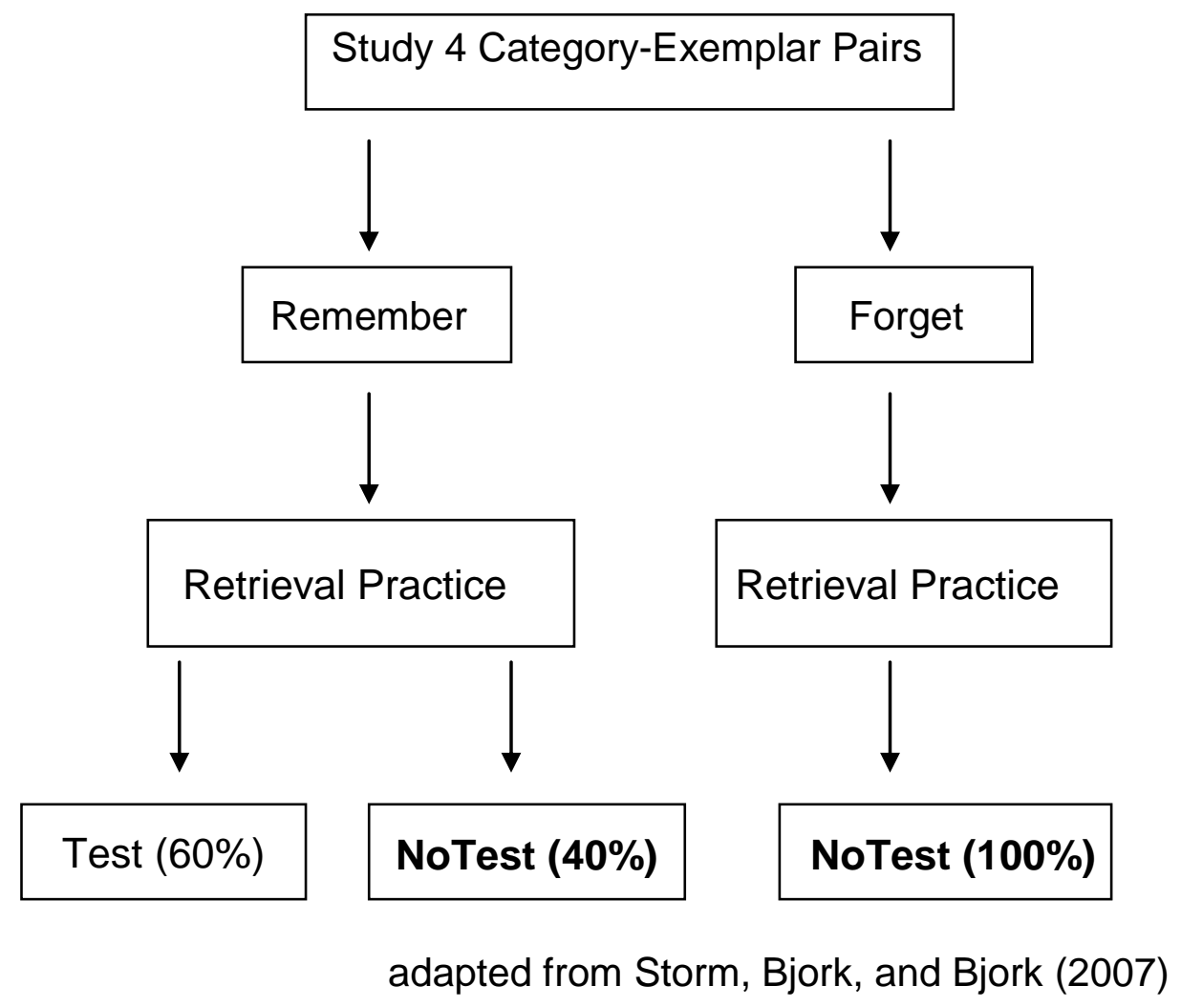

Figure 1. Diagram of trial sequence. 


\section{PROBLEM}

\subsection{Testing for Inhibitory Control in Creative Problem Solving}

If problem-solving-induced forgetting is caused by a similar inhibitory mechanism that acts to resolve competition from interfering items during creative problem solving, similar to the results of Storm et al. (2007), forgetting should be found following problem solving only for the interfering items that participants are instructed to remember, and not for the interfering items that participants are instructed to forget, prior to problem solving. Alternatively, if problem-solving-induced forgetting is not the result of inhibition, forgetting should be found following problem solving for interfering items participants are instructed to remember and forget.

Manipulating the competitiveness of incorrect associates prior to RAT problem solving using remember and forget instructions and a procedure very similar to that of Storm et al. (2007), this study aims to test for inhibition as an explanation for the problem-solving-induced forgetting reported in Storm et al. (in press). Manipulating the competitiveness of incorrect associates will also affect the degree of fixation induced during problem solving. If inhibitory control acts to overcome fixation, more forgetting should also be found for incorrect associates participants are instructed to remember because they should be more likely to block problem solving and induce fixation, when compared to the incorrect associates that participants are instructed to forget. However, if problem-solving-induced forgetting is not the result of an active process to overcome 
fixation and is a by-product of solving RAT problems, more forgetting should be found for incorrect associates participants are instructed to remember and forget.

When participants are presented with impossible RAT problems, for which there is no correct solution, the amount of problem-solving-induced forgetting following short and long problem solving durations can be compared (Storm et al., in press). Storm et al. (in press) found increased forgetting for incorrect associates following longer durations of impossible RAT problem solving. In addition to replicating this result, the current study aims to further investigate this finding. If problem-solving-induced forgetting is the result of inhibitory control resolving competition and overcoming fixation, the greatest amount of forgetting should occur for incorrect associates participants are instructed to remember following long problem solving durations (see Figure 2). An opposite prediction exists, however, based on the argument that the longer a person remains fixated the more difficult it becomes to overcome the fixation, resulting in entrenchment (Moss, Kotovsky, \& Cagan, 2011). According to this entrenched fixation account and if problem-solving-induced forgetting is simply the result of noninhibitory retrieval biases experienced during the final test that accumulate during problem solving and become stronger with longer problem solving durations, greater forgetting should occur for incorrect associates participants are instructed to remember and forget following long problem solving durations compared to forgetting following short problem solving durations (see Figure 3). 
Instructions Prior to Problem Solving
Remember
Forget

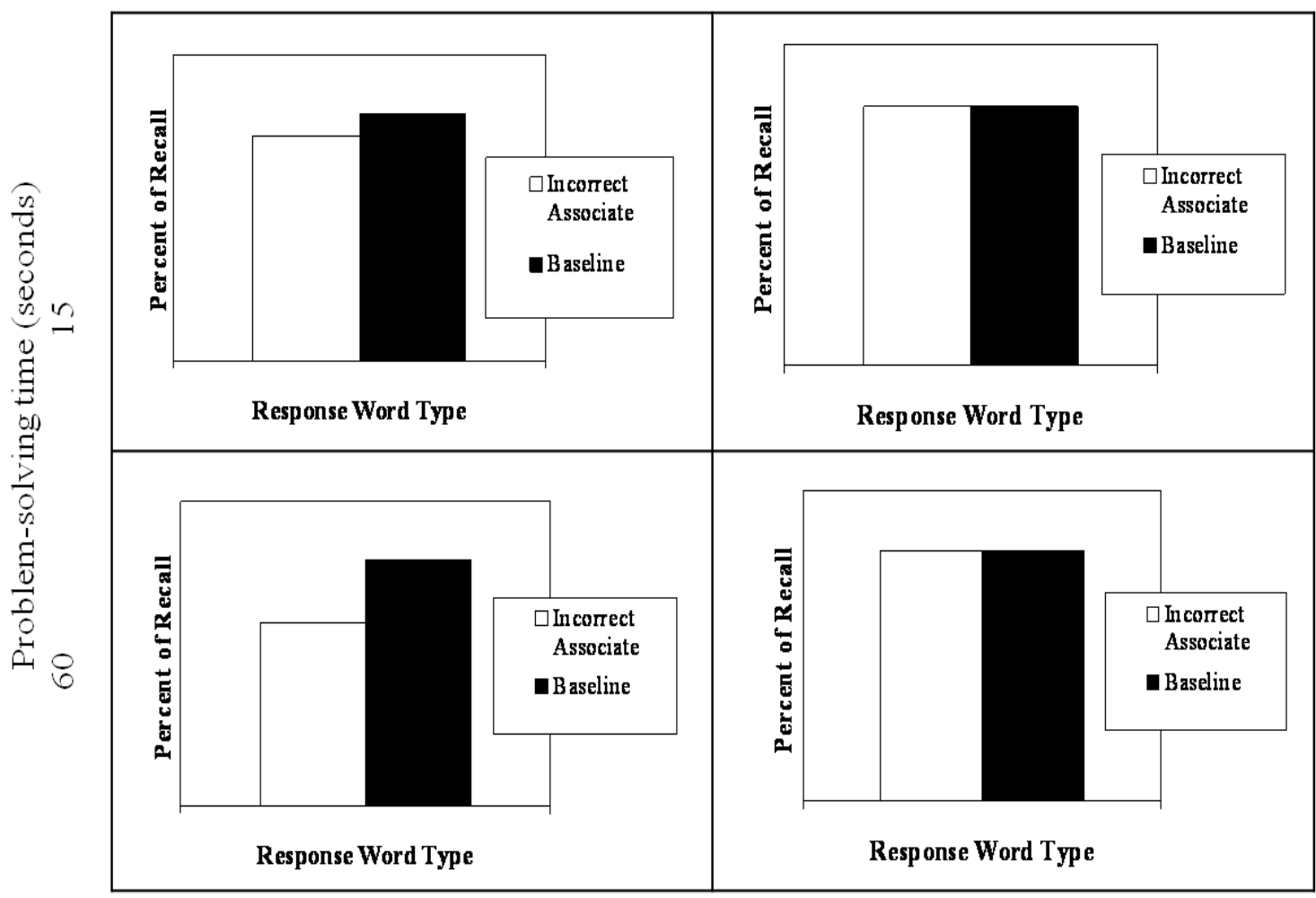

Figure 2. Predicted results using inhibitory control explanation. 
Instructions Prior to Problem Solving

Remember

Forget

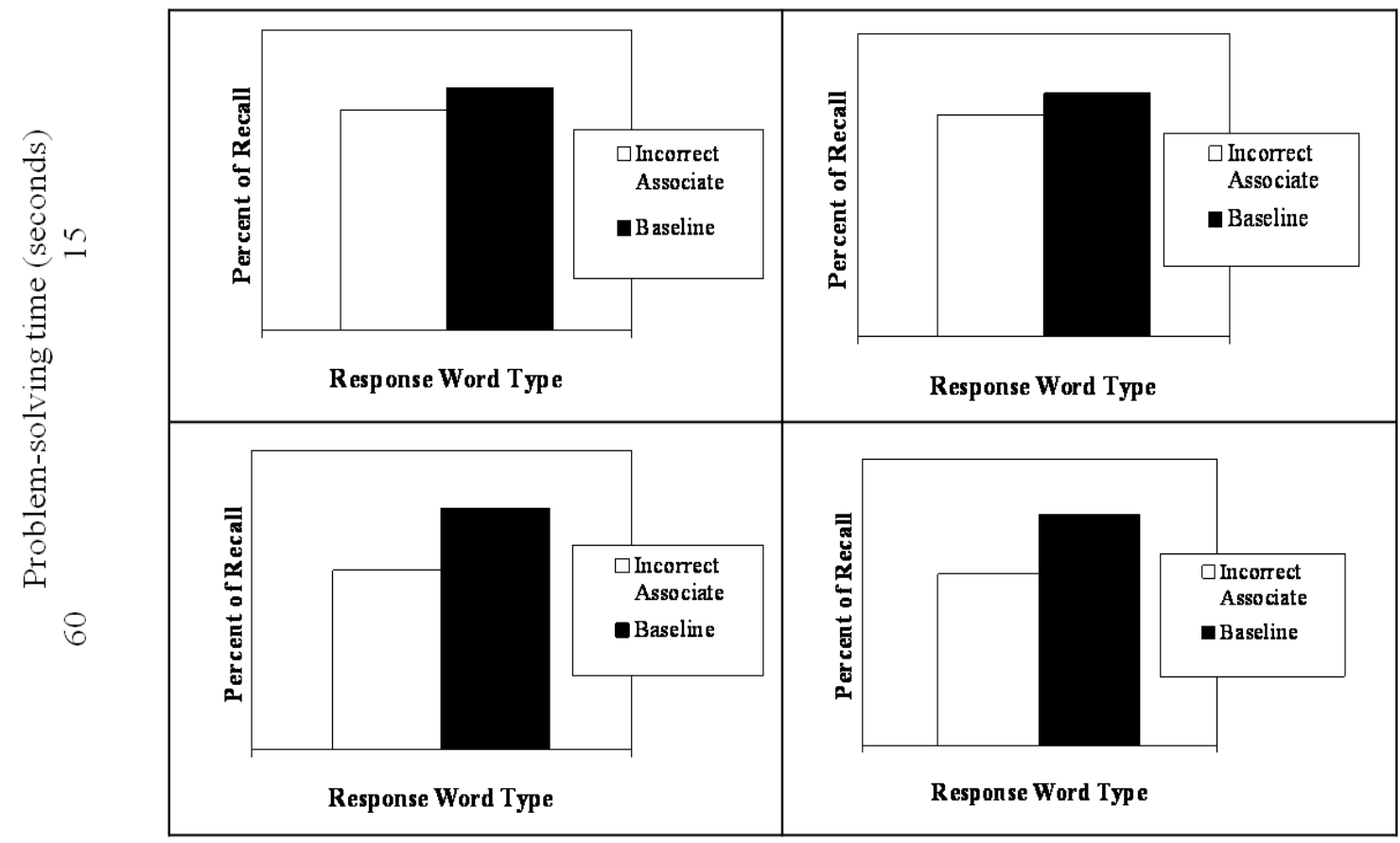

Figure 3. Predicted results using non-inhibitory explanation.

\subsection{Method}

Participants. Fifty-six Texas A\&M undergraduates (30 males, 26 females; mean age $=19.4)$ participated for partial credit in an introductory psychology course. Students participated individually in a randomly assigned counterbalanced condition.

Materials. Twelve RAT problems with possible solutions were presented. Six RATs were written by Mednick and Mednick (1967), with an average of $42 \%$ of previously tested participants successfully solving these problems after five seconds 
(Shames, 1994). The remaining six RATs were written by Bowers, Regehr, Balthazard, and Parker (1990), with an average of 55\% of previously tested participants solving these problems after five seconds (Shames, 1994). Twelve additional possible problems were taken from those written by Bowden and Jung-Beeman (2003), with an average of $27 \%$ of their tested participants solving these problems after seven seconds. Seventytwo associated cue-response word pairs were created using each of the RAT problem words as cues and a unique selected response word with moderate forward associative strength, with the requirement that the response word was never a problem's answer or a problem word for a different problem. All 56 participants studied each of the 72 word pairs, but only 36 of the word pairs involved a cue word that was also a presented problem word.

Ninety-six problem words were selected from 57 additional problems written by Bowden and Jung-Beeman (2003). These words were combined to form 32 impossible RAT problems, with the requirement that none of the impossible problems had two or more words taken from the same original problem. Half of the participants were given 16 of the 32 impossible problems to solve, while the remaining 28 participants were given the remaining 16 impossible problems. Ninety-six associated cue-response word pairs were created using each of the impossible problem words as cues and a unique selected response word with moderate forward associative strength, with the requirement that the response word was never a RAT problem's answer or a problem word for a different problem. All 56 participants studied each of the 96 word pairs, but only 48 of the word pairs involved a cue word that was also a presented impossible problem word. 
These critical pairs contained the incorrect associates, for which forgetting was tested following problem solving. The remaining 48 associated response words served as baseline words for final cured-recall performance.

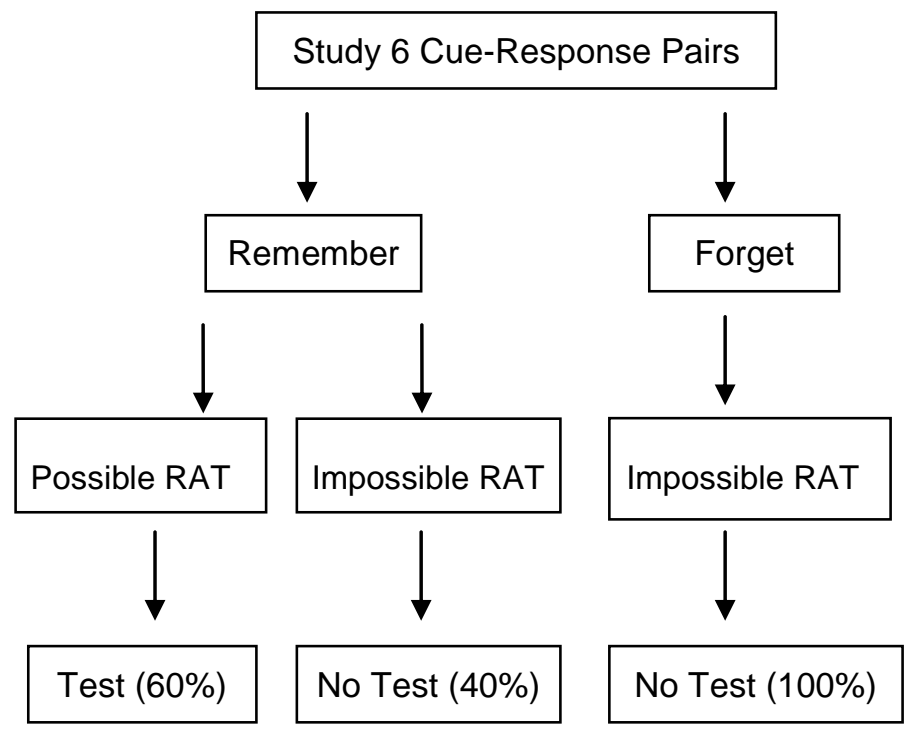

Figure 4. Sequence of current study's trials.

Procedure. Each participant received 12 trials, see Figure 4, with four phases: cue-response study, Remember instructions for studied pairs, possible RAT problem solving, and an expected cued-recall test for the associated response words. Of these 12 trials, six included RATs that were each presented for 15 seconds, while the remaining six were each presented for 60 seconds. Participants also received 16 trials with three phases: cue-response study, Remember or Forget instructions for studied pairs, and impossible RAT problem solving. Of these 16 trials, eight included Remember 
instructions, while the remaining eight included Forget instructions. Additionally, eight impossible problems were presented for 15 seconds, and the remaining eight were presented for 60 seconds. Impossible problem presentation time was crossed with type of instructions for the studied pairs, creating four different impossible problem solving conditions. Both factors were counterbalanced between subjects so that each of the 32 impossible problems followed each instruction type and was shown for each presentation time. The 28 trials (12 possible and 16 impossible) were presented in a set random order so that a participant could never predict the type of instructions for studied pairs nor the presentation time of a given possible or impossible RAT problem. Participants were given a number shadowing task for a five-digit number after every trial to reduce proactive interference on subsequent trials. Once all 28 trials had been completed, the participant was given a distractor task for 5 minutes, followed by a surprise cued-recall test for all of the cue-response word pairs that had been presented during the experiment.

Cue-response study. Six cue-response word pairs were presented together on the computer screen for 12 seconds. Participants were instructed to spend that time relating the response word to the cue word so that if they were shown the cue word, they would be able to produce the response word. Each word pair was offset on the screen to deter participants from creating associations between words from different pairs. Three of the presented cue words were also problem words for the subsequently presented RAT during the trial, while the remaining three cue words were never presented as problem words for that participant. 
Remember instructions for studied pairs. For 20 of the 28 trials, immediately following the study phase, participants were given instructions to remember the previously presented word pairs because they would probably take a memory test for the response words after they solved a word problem. Participants were informed that this test would occur $60 \%$ of the time that they saw Remember instructions. This resulted in 12 trials with expected cued-recall tests and eight trials that received remember instructions and no actual expected cued-recall test.

Forget instructions for studied pairs. For eight of the 28 trials, immediately following the study phase, participants were given instructions to forget the previously presented word pairs because they would not take a memory test for the response words after they solved the word problem. Participants were informed that a test would occur $0 \%$ of the time that they saw Forget instructions.

Problem solving. Participants attempted to solve a possible or an impossible RAT problem. However, the participants were uninformed regarding the type of problem, as well as the existence of impossible RAT problems. Participants were given instructions that, for each trial, the solution word could be related in meaning to a problem word, share the same meaning as the problem word, or could combine with the problem word to form a common English phrase. They were also informed that any of these relations would lead to possible solutions, with the requirement that the solution word must be related in some way with each of the three problem words. These instructions ensured that solution success would require multiple attempts, creating greater likelihood that competition from previously studied word pairs would need to be 
resolved. Most importantly, participants were warned that none of the previously studied response words would be problem solutions. Participants were encouraged to continue trying to solve the problem while it remained presented on the screen. Each verbal response was recorded, and participants received feedback for their inaccuracy each time an incorrect response was made. Impossible RAT trials ended once the problem left the screen. Participants then completed the number shadowing task prior to the beginning of the next trial. When they were successful at generating the solution during possible RAT trials, or when they were unsuccessful but the problem presentation time had elapsed, they moved on to the expected cued-recall test for the studied word pairs.

Expected cued-recall test. Each of the given trial's six initially studied cue words were presented on the screen in the original studied locations followed by a blank line of uniform length replacing the six associated response words. Participants were given 18 seconds to produce all six of the missing response words. They were free to provide the response words in any order, with the requirement that they point to the cue word while they generate the response word. Each verbal response was recorded by the experimenter.

Surprise cued-recall test. The 96 cue words that were studied prior to impossible RAT problem solving were presented in a random set order and participants were given four seconds to recall each response word. Without a break in presentation, the remaining 72 cue words that were studied prior to possible RAT problem solving were then presented in a random set order for four seconds each and participants continued to 
recall the associated response word. Prior to the test, participants were told that they should respond only with the response word that they had studied with each cue word. They were also instructed that they would need to provide all of the previously studied response words, even if they had received instructions to forget the response words during the experiment. 


\section{RESULTS AND DISCUSSION}

\subsection{Overall Results}

A 2 (incorrect associate vs. baseline item) x 2 (15 vs. 60 second problem solving) x 2 (remember vs. forget) repeated measures analysis of variance was performed on the surprise cued-recall results only for items presented during impossible RAT trials, which were also items never tested with the expected cued-recall test. Overall, problemsolving-induced forgetting occurred (see Figure 5) because the incorrect associates of impossible problems were recalled less often $(M=0.30, S D=0.18)$ than baseline items $(M=0.34, S D=0.18), F(1,55)=18.85, p<0.05$. Studied items, including baseline and incorrect associates, were not recalled less often when they were presented before 60 s of problem solving $(M=0.30, S D=0.18)$ when compared to these items' recall when presented before $15 \mathrm{~s}$ of problem solving $(M=0.31, S D=0.18), F(1,55)=0.034, p=$ 0.86. The studied items, both baseline and incorrect associates, were also not recalled less often when they were given remember instructions $(M=0.31, S D=0.18)$ compared to when they were given forget instructions $(M=0.30, S D=0.18), F(1,55)=0.087, p=$ 0.77. The predicted three-way interaction based on the inhibitory control explanation between item type, problem solving duration, and type of instructions was not significant, $F(1,55)=0.156, p=0.69$. Similarly, the predicted two-way interaction based on the inhibitory control explanation between item type and type of instructions was not significant, $F(1,55)=0.728, p=0.40$. The predicted two-way interaction based 
on the non-inhibitory explanation between item type and problem solving duration was also not significant, $F(1,55)=1.39, p=0.24$.

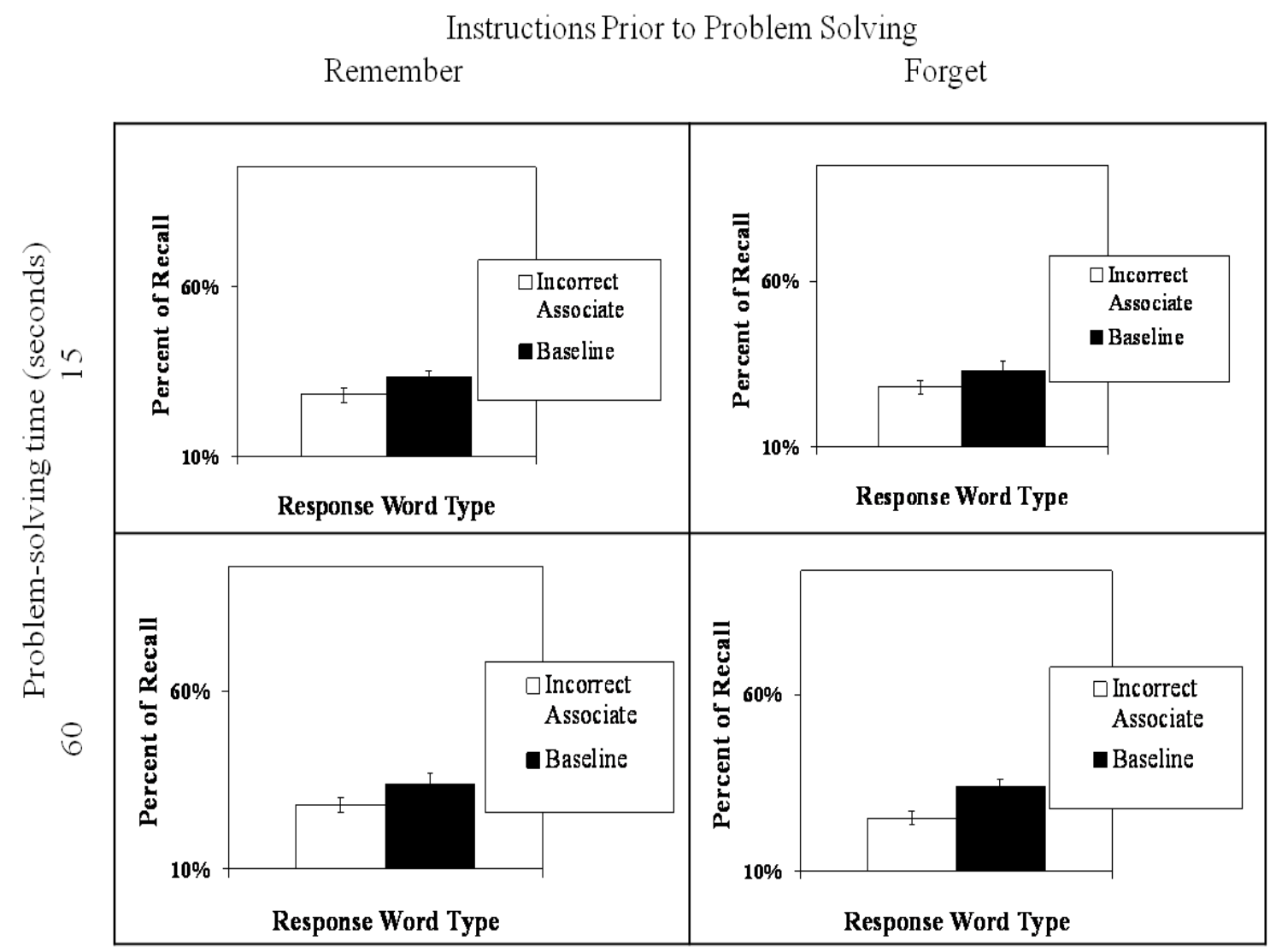

Figure 5. Overall results separated by within-subjects condition.

One possible reason for the failure to replicate the results of Storm et al. (2007) is that a floor effect occurred when measuring final recall performance. With such low rates of recall for baseline items, it might be difficult to measure different rates of 
problem-solving-induced forgetting for incorrect associates given remember or forget instructions and studied before $15 \mathrm{~s}$ or 60 s problem solving. The reduced levels of recall for baseline items could have occurred because of the longer retention interval between study and final test used in this study compared to the retention interval used by Storm et al. This study included 28 total trials, each presenting 6 word pairs for study. When compared to the 7 trials with retrieval practice rather than impossible RAT problem solving, as well as with only 4 studied word pairs per trial, used by Storm et al., this study's procedure presents much more difficulty for recalling baseline items during the

final surprise cued-recall test. In fact, overall, the baseline response words were recalled less often $(M=0.34, S D=0.18)$ when compared with the baseline exemplars reported by Storm et al. $(M=0.55, S D=0.30)$. Secondly, the increased number of challenging trials in this study presents more potential for participants to experience fatigue or refuse to continue to follow remember and forget instructions.

\subsection{Exploratory Analysis}

Exploratory analyses were carried out to compare the final recall rates for incorrect associates and baselines items presented during the first, second, third, and fourth quartiles of experiment trials. Each quartile included six cue-response pairs that were given remember or forget instructions, three with incorrect associates that were each cued with a RAT problem word for the subsequently presented impossible RAT problem and the remaining three including baseline response words, prior to either 15 or 60 
seconds of impossible RAT problem solving, resulting in one set of six pairs for each of the four possible within-subjects conditions. According to a paired-samples t-test performed on the recall results for response words presented during the first quartile (see Figure 6), incorrect associates that participants were instructed to remember prior to 60 seconds of problem solving were recalled less often $(M=0.18, S D=0.26)$ when compared with recall of baseline items $(M=0.29, S D=0.30), t(55)=-2.045, p<0.05$. None of the other paired-samples tests for recall data from the first quartile were significant. Interestingly, this particular trend was not found for the second, third, or fourth quartiles. 
Instructions Prior to Problem Solving

Remember

Forget

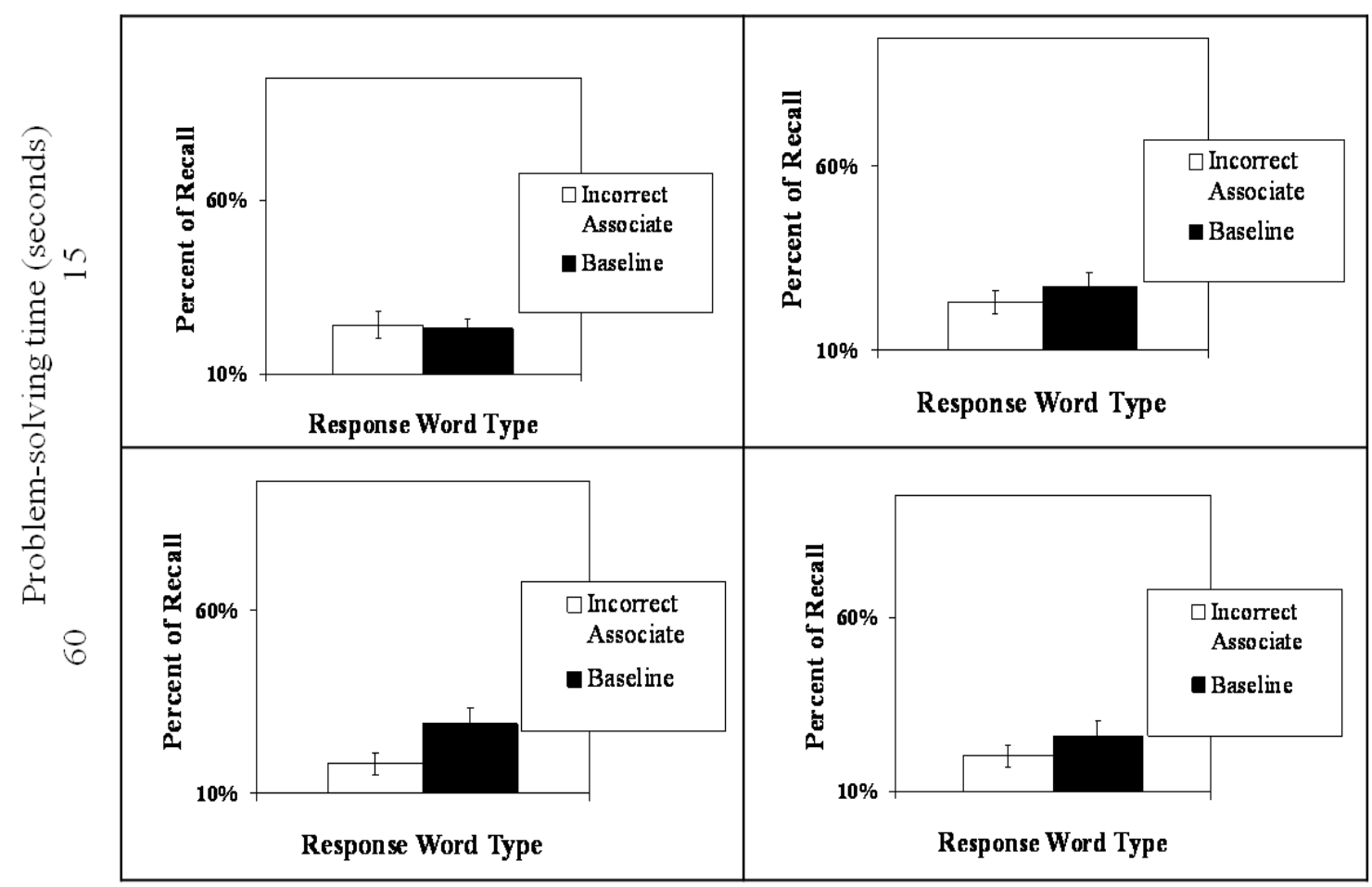

Figure 6. First quartile results separated by within-subjects condition. 


\section{GENERAL DISCUSSION}

Overall, problem-solving-induced forgetting occurred for incorrect associates that presumably interfered during problem solving and blocked solvers from generating additional associates in attempts to find a solution. However, because problem-solvinginduced forgetting occurred for both the incorrect associates participants were instructed to remember and those they were instructed to forget, the results do not support the inhibitory control explanation. Problem-solving-induced forgetting took place even for the incorrect associates participants were told to forget. These associates, however, should not have interfered or competed for access during subsequent problem solving and, therefore, should not have been subject to inhibitory control and targeted suppression. Additionally, the results do not support the non-inhibitory explanation of problem-solving-induced forgetting. According to this explanation, forgetting occurs for incorrect associates that are cued at the final test with RAT problem words because of reduced accessibility at test caused by biases that developed and grew during problem solving. For example, an associative bias could have been created for other associates generated during problem solving so that later, during the final cued-recall test, the RAT

problem word is more likely to cue associates generated during problem solving and less likely to cue the incorrect associates that were initially studied with the RAT problem words. If problem-solving-induced forgetting is caused by biases created during problem solving when new associates are generated, more forgetting should occur following longer problem solving durations, regardless of whether participants are 
instructed to remember or forget the previously studied incorrect associates. However this result was not found. Overall problem-solving-induced forgetting did not increase with longer problem solving durations.

Although the results failed to support either inhibitory control or non-inhibitory explanations for problem-solving-induced forgetting, the exploratory analysis of problem-solving-induced forgetting during the first quartile of trials suggests a potential role of inhibitory control that will need to be tested further with a follow-up study with less experiment trials. In fact, a study with fewer trials will be more comparable to Storm et al. (2007) and will possibly result in better baseline recall on the final cuedrecall test, which may reveal different patterns of problem-solving-induced forgetting. A follow-up study with fewer trials will present fewer total response words for study and later test. In addition, a study with fewer trials will also have a much shorter retention interval between initial study and final test. Fewer tested response words and a shorter retention interval should lead to improved recall for baseline response words, which will provide a more accurate comparison group for observing the recall deficits caused by problem solving. If a study with fewer trials results in problem-solving-induced forgetting only for the incorrect associates that participants were instructed to remember following 60 seconds of problem solving, as was observed during the first quartile, the results will provide evidence for inhibitory control in suppressing blocks in creative problem solving.

It seems likely that creative problem solving success must involve a filtering process that includes relevant background knowledge and excludes irrelevant knowledge 
that competes for access. This exclusion, however, requires active participation from the problem solver. The fact that a trend for problem-solving-induced forgetting occurred for incorrect associates participants were instructed to remember only for longer periods of problem solving during the first quartile of study trials suggests that the exclusion and inhibition of interfering information is not automatic and depends on the amount of time spent attempting to solve the problem. However, additional problem solving time can also lead to a greater number of generated responses; which, according to the noninhibitory explanation, can act to bias the RAT problem word retrieval cue during the final test and cause forgetting for the studied incorrect associates. However, Storm et al. (in press) found no correlation between the total number of responses generated during impossible RAT problem solving and the amount of problem-solving-induced forgetting those impossible RAT problems caused. Future work will need to further investigate the role of solution attempts in problem-solving-induced forgetting.

One technique used in creative problem solving is analogical transfer, the process of applying an analogous product or solution to a problem to produce a new product or solution (e.g., Dahl \& Moreau, 2002). In this case, the problem's mental representation is either added to or chiseled within the framework of the governing analogy without a complete restructuring. While applying a current mental framework or solution towards a problem can assist productive thinking, it can also create a new challenge. Now a successful solution requires including only details of the analogy that are relevant to the problem and excluding all other irrelevant ones. According to Dominowski (1995), although it is unnecessary for the creative problem solver to have expert knowledge 
within the problem's domain, the solver must be able to shift his or her perspective in order to see the problem in a different way. Additionally, he argues, the perceptual presentation of the problem exerts strong influence on the initial perspective of the problem, which leads to typical incorrect solutions. In order to change the way the problem is viewed and mentally structured, the solver must be able to overcome the problem's perceptual influence. This skill, Dominowski explains, is also associated with other types of creative production. Restructuring the problem, however, would become easier following the passage of time or changing the context in which the problem is presented. Therefore, there may be ways to anticipate blocks in creative thinking, and to protect oneself from those blocks by switching to a different context, perhaps by explaining the problem to someone else, or returning to the problem later. As the pattern of results of this study might suggest, however, a more active approach to fighting blocks in creative thinking would be to target and suppress unwanted and interfering information that comes to mind.

Two types of information that tend to cause fixation are recent experience and implicit assumptions, which can interact to create conformity effects (Smith, 2003). Such effects have been observed when participants generate ideas that include more qualities of presented examples when compared with the qualities included in generated ideas of participants that never see examples, even when participants are instructed to avoid producing ideas similar to the examples (e.g., Jansson \& Smith, 1991; Smith, Ward, \& Schumacher, 1993). It could be possible that participants are unaware of the influences that the presented examples seemed to be exerting on their work. This would 
explain why an instruction to avoid conforming to the examples would be ineffective because it is not possible to avoid something of which you are unaware. Similarly, improvement in RAT problem solving following induced fixation occurs only when participants are aware of the interfering incorrect associates that were blocking them from accessing the solution (e.g., Vul \& Pashler, 2007). Future work will need to investigate the role of awareness in overcoming fixation with inhibitory control.

A mechanism that can exclude irrelevant details of an analogy, interfering background knowledge, or inappropriate features of the mental set applied to a creative problem is essential, not only, for producing a solution, but also for avoiding time wasted on bad solutions. Storm et al. (in press) found a positive correlation between the amount of problem-solving-induced forgetting following impossible RAT problem solving and the number of possible RAT problems solved following induced fixation. These correlational results, when combined with the trend found in this study, suggest that inhibition of unwanted information during problem solving might be one mechanism for preventing mental fixation.

The existence of inhibitory control, which enables people to target interfering and incorrect items during creative problem solving, has practical importance for fields prone to biases from recent experience and implicit assumptions such as engineering design (Jansson \& Smith, 1991) and medical pathology (Croskerry, 2003). If interfering information from recent experience or implicit assumptions can be suppressed during problem solving, increased time spent working towards a creative solution should result in reduced biases and less fixation. This prediction, however, counters the concept of 
entrenched fixation, which according to Moss et al. (2011) suggests that once a person has fixated onto an incorrect response they will be led further away from the problem's solution with increased time spent problem solving. If future work shows evidence for inhibitory control resulting in greater forgetting for incorrect competing responses with increased time spent problem solving, creative problem solvers such as engineers and pathologists might benefit from increased problem solving intervals, especially those that encourage problem solving past the point of fixation and mental blocks. 


\section{CONCLUSION}

This study provided additional evidence for problem-solving-induced forgetting. Participants showed impaired recall performance for incorrect associates of RAT problem words following problem solving, when compared with baseline associates of words that were never presented during problem solving as RAT problem words. Overall, however, these baseline associates were recalled less often when compared with the baseline recall rate of Storm et al. (2007), which used a similar design and procedure, except with less items and a shorter retention interval between study and final cuedrecall test. When results were analyzed by the first, second, third, and fourth quartile of experiment trials, only the incorrect associates studied during the first quartile that participants were instructed to remember showed problem-solving-induced forgetting following the longer, but not shorter, problem solving duration. This trend was only observed during the first quartile of trials, which had the fewest number of study items, along with less potential for fatigue or frustration from impossible RAT problem solving and following remember and forget instructions. Future work will need to investigate this trend with a study using fewer items studied and later tested, as well as a shorter retention interval between study and test. The replication of this trend will provide support for the inhibitory-control explanation of problem-solving-induced forgetting suggesting that people can suppress incorrect and interfering information that competes for retrieval access during creative problem solving, resulting in inhibition of unwanted items. 


\section{REFERENCES}

Anderson, M.C. (2003). Rethinking interference theory: Executive control and the mechanisms of forgetting. Journal of Memory and Language, 49, 415-445.

Anderson, M.C. (2005). The role of inhibitory control in forgetting unwanted memories: A consideration of three methods. In C. MacLeod \& B. Uttl (Eds.), Dynamic cognitive processes (pp. 159-190). Tokyo: Springer-Verlag.

Anderson, M. C., \& Bjork, R. A. (1994). Mechanisms of inhibition in long-term memory: A new taxonomy. In D. Dagenbach \& T. Carr (Eds.), Inhibitory processes in attention, memory, and language (pp. 265-325). New York: Academic Press.

Anderson, M.C., Bjork, R.A., \& Bjork, E.L. (1994). Remembering can cause forgetting: Retrieval dynamics in long-term memory. Journal of Experimental Psychology: Learning, Memory, and Cognition, 20, 1063-1087.

Anderson, M.C., \& Spellman, B.A. (1995). On the status of inhibitory mechanisms in cognition: Memory retrieval as a model case. Psychological Review, 102, 68100.

Bjork, E. L., Bjork, R. A., \& Anderson, M. C. (1998). Varieties of goal-directed forgetting. In J. M. Golding and C. MacLeod (Eds.), Intentional forgetting: Interdisciplinary approaches (pp.103-137). Hillsdale, NJ: Erlbaum.

Bjork, R. A. (1970). Positive forgetting: The noninterference of items intentionally forgotten. Journal of Verbal Learning and Verbal Behavior, 9, 255-268. 
Bjork, R. A. (1989). Retrieval inhibition as an adaptive mechanism in human memory. In H. L. Roediger and F. I. M. Craik (Eds.), Varieties of memory and consciousness: Essays in honour of Endel Tulving (pp. 309-330). Hillsdale, NJ: Erlbaum.

Bowden, E.M. and Jung-Beeman, M. (2003) Normative data for 144 compound remote associate problems. Behavioral Research Methods, Instruments, \& Computers, 35, 634-639.

Bowers, K.S., Regehr, G, Balthazad, C.G., \& Parker, K. (1990). Intuition in the context of discovery. Cognitive Psychology, 22, 72-110.

Croskerry, P. (2003). The importance of cognitive errors in diagnosis and strategies to minimize them. Academic Medicine, 78(8), 775-780.

Dahl, D.W., \& Moreau, P. (2002). The influence and value of analogical thinking during new product ideation. Journal of Marketing Research, 39(1), 47-60.

Dominowski, R.L. (1995). Productive problem solving. In S.M. Smith, T.B. Ward, \& R.A. Finke (Eds.) The creative cognition approach (pp. 73-95). Cambridge, MA: MIT Press.

Gelfand, H., \& Bjork, R.A. (1985, November). On the locus of retrieval inhibition in directed forgetting. Paper presented at the meeting of the Psychonomic Society, Boston, MA.

Jansson, D. G., \& Smith, S. M. (1991). Design fixation. Design Studies, 12 (1), 3-11.

McGeoch, J.A. (1942). The psychology of human learning. New York: Longmans, Green. 
Mednick, S.A. (1962). The associative basis of the creative process. Psychological Review, 69, 220-232.

Mednick, S. A. \& Mednick, M. T. (1967). Examiner's manual: Remote Associates Test. Boston: Houghton Mifflin.

Moss, J., Kotovsky, K., \& Cagan, J. (2011). The effect of incidental hints when problems are suspended before, during, or after an impasse. Journal of Experimental Psychology: Learning, Memory, and Cognition, 37 (1), 140-148.

Postman, L., Stark, K., \& Fraser, J. (1968). Temporal changes in interference. Journal of Verbal Learning and Verbal Behavior, 7, 672-694.

Shames, V.A. (1994). Is there such a thing as implicit problem-solving? Unpublished doctoral dissertation, University of Arizona, Tucson.

Smith, S. M. (2003). The constraining effects of initial ideas. In P. Paulus and B. Nijstad (Eds.) Group creativity: Innovation through collaboration (pp. 15-31). Oxford, England: Oxford University Press.

Smith, S. M., \& Blankenship, S. E. (1989). Incubation effects. Bulletin of the Psychonomic Society, 27, 311-314.

Smith, S. M., \& Blankenship, S. E. (1991). Incubation and the persistence of fixation in problem solving. American Journal of Psychology, 104, 61-87.

Smith, S. M., \& Vela, E. (1991). Incubated reminiscence effects. Memory \& Cognition, 19(2), 168-176.

Smith, S.M., Ward, T.B., \& Schumacher, J.S. (1993). Constraining effects of examples in a creative generation task. Memory \& Cognition, 21, 837-845. 
Storm, B. C. (2011). Retrieval-induced forgetting and the resolution of competition. In A.S. Benjamin (Ed.), Successful remembering and successful forgetting: A Festschrift in honor of Robert A. Bjork (pp. 89-105). New York, NY: Psychology Press.

Storm, B.C., \& Angello, G. (2010). Overcoming fixation: Creative problem solving and retrieval-induced forgetting. Psychological Science, 21, 1263-1265.

Storm, B.C., Angello, G., \& Bjork, E.L. (2011). Thinking can cause forgetting: Memory dynamics in creative problem solving. Journal of Experimental Psychology: Learning, Memory, and Cognition. Advance online publication. doi: $10.1037 / \mathrm{a} 0023921$

Storm, B. C., Bjork, E. L., \& Bjork, R. A. (2007). When intended remembering leads to unintended forgetting. Quarterly Journal of Experimental Psychology, 60, 909915.

Vul, E., \& Pashler, H. (2007). Incubation benefits only after people have been misdirected. Memory \& Cognition, 35, 701-710.

Weisberg, R.W. (1995). Case studies of creative thinking: Reproduction versus restructuring in the real world. In S.M. Smith, T.B. Ward, \& R.A. Finke (Eds.) The creative cognition approach (pp. 53-72). Cambridge: MIT Press.

Wiley, J. (1998). Expertise as mental set: The effects of domain knowledge in creative problem solving. Memory \& Cognition, 26, 716-730. 


\section{VITA}

Name: Genna Marie Angello

Address: $\quad$ Texas A\&M University, 4235 TAMU,

College Station, TX 77843-4235

Email Address: gennaangello@tamu.edu

Education: $\quad$ B.A., Psychology, University of California, Los Angeles, 2008

M.S., Psychology, Texas A\&M University, 2011 